\section{Adjustable acuity}

A study of monocular deprivation (MD) in rats reveals an enhancement of visual acuity through the nondeprived eye after long periods of MD. Moreover, a subsequent period of eye opening and reclosing suggests that there is a considerable plasticity of visual function into adulthood.

Brief periods of MD, or MD commenced in adulthood, typically have no long-term effects on visual function. However, if MD is commenced during early postnatal life and prolonged into adulthood there are pronounced effects on the function of the visual cortex, with most visual cortex neurons no longer being excited by input to the deprived eye, but only by input to the non-deprived eye. Unsurprisingly, this change is accompanied by an impairment of visually guided behaviour through the deprived eye. Recently, electrophysiological recordings of the mouse visual cortex showed that MD results not only in a depression of visually evoked potentials through the deprived eye, but also an enhancement of visually evoked potentials through the nondeprived eye. However, it was uncertain whether the latter effect was associated with a change in visual function in the non-deprived eye.

Mark Bear and colleagues assessed the behavioural significance of these findings by measuring visual acuity in rats after five months of MD that began at postnatal day 21 . As expected, MD resulted in a decrease in visual acuity through the deprived eye; in addition, however, MD also produced an enhancement of visual acuity over and above normal levels through the non-deprived eye. This suggests that the previously described changes in visually evoked potentials following MD are functionally relevant.

Following the opening of the deprived eye, and restoration of binocular vision, there was a

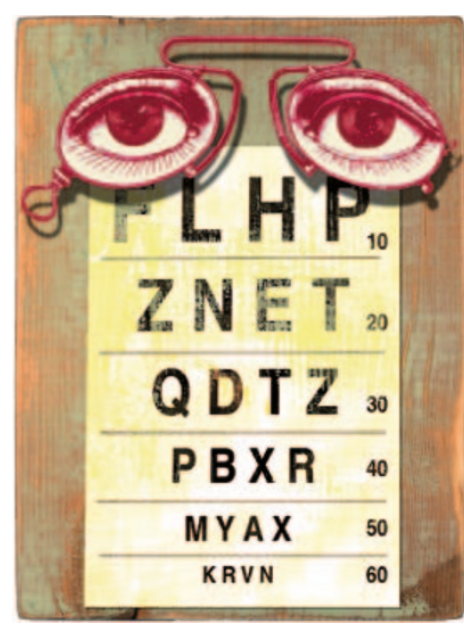

reduction to near-normal levels of visual acuity through the nondeprived eye, which was followed by a gradual but incomplete recovery of acuity through the deprived eye. To determine whether this plasticity of visual function was reversible and preserved in adulthood, animals were then subjected to a second period of MD. Visual acuity through the nondeprived eye increased once more to the same enhanced level seen after the initial period of MD. When binocular vision was again restored, acuity through the non-deprived eye declined once more, whereas acuity through the deprived eye, which had decreased during the period of MD, recovered with time.

These findings show a robust and reversible plasticity in the visual function of rats that extends well into adulthood. It will be interesting to elucidate the physiological and molecular mechanisms responsible for these changes in visual performance and how these might contribute to enhanced vision or 'lazy eye' syndrome in humans.

Daniel McGowan

ORIGINAL RESEARCH PAPER Iny, K. et al. Bidirectional modifications of visual acuity induced by monocular deprivation in juvenile and adult rats. J. Neurosci. 26, 7368-7374 (2006)

\section{IN BRIEF}

\section{$\Rightarrow$ SYNAPTIC PHYSIOLOGY}

Antagonistic regulation of synaptic vesicle priming by Tomosyn and UNC-13.

McEwen, J. M. et al. Neuron 51, 303-315 (2006)

Neurotransmitter release requires the priming of synaptic vesicles by an interaction between vesicle and plasma membrane proteins to form a SNARE complex. McEwen and colleagues show that formation of the SNARE complex depends on balancing the opposite actions of two SNARE-interacting proteins: tomosyn, which inhibits SNARE complex formation, and UNC13, which promotes this process. Furthermore, they show an additional and unexpected post-priming function for UNC13 in promoting stimulus-evoked synaptic vesicle fusion.

\section{$\Rightarrow$ DEVELOPMENT}

DIG-1, a novel giant protein, non-autonomously mediates maintenance of nervous system architecture.

Benard, C. Y. et al. Development 133, 3329-3340 (2006)

Dedicated maintenance mechanisms that preserve the complex architecture of the nervous system once patterning is complete are now emerging. Using Caenorhabditis elegans, Benard and colleagues show that DIG1 - a giant secreted protein produced by muscle cells after the completion of development - maintains the axonal position of the ventral nerve cord and cell body positioning in ganglia of the head, probably by stabilizing the basement membrane.

\section{SENSORY SYSTEMS}

A second class of chemosensory receptors in the olfactory epithelium.

Liberles, S. D. \& Buck, L. B. Nature 30 July 2006 (doi:10.1038/ nature05066)

The 1,000 or so members of the odorant receptor family found on olfactory sensory neurons (OSNs) allow the discrimination of a large variety of odorants. However, some OSNs are stimulated by small peptides and pheromones, which are not ligands for odorant receptors, suggesting the presence of additional classes of receptor. A newly identified class of chemosensory receptors, expressed in a small population of OSNs that seem to lack odorant receptors, has now been described. Called trace amineassociated receptors, these receptors, which are evolutionarily conserved, appear to recognize social cues such as pheromones.

\section{$\Rightarrow$ BEHAVIOURAL NEUROSCIENCE}

Cortical $5-\mathrm{HT}_{2 \mathrm{~A}}$ receptor signalling modulates anxietylike behaviours in mice.

Weisstaub, N. V. et al. Science 313, 536-540 (2006)

Altered serotonin (5-HT) signalling has been implicated in disorders of both anxiety and depression. The $5-\mathrm{HT}_{2 \mathrm{~A}}$ receptor $\left(5-H T_{2 A} \mathrm{R}\right)$ is just one of many serotonin receptors, but its high expression level in the cortex, ventral striatum, hippocampus and amygdala - regions associated with anxiety-like behaviours suggested that it might mediate the effects of serotonin on these behaviours. Weisstaub et al. show that mice deficient in $5-\mathrm{HT}_{2 \mathrm{~A}} \mathrm{R}$ display decreased signs of conflict anxiety, and that selective restoration of $5-\mathrm{HT}_{2 \mathrm{~A}} \mathrm{R}$ in the forebrain normalized anxietyrelated behaviour. Fear and depression-related behaviours were unaffected, suggesting a specific role for cortical $5-\mathrm{HT}_{2 \mathrm{~A}} \mathrm{R}$ in modulating conflict anxiety. 